A) Check for updates

Cite this: Nanoscale, 2020, 12, 6529

\section{Rational construction of a triple emission molecular imprinting sensor for accurate naked- eye detection of folic acid $\dagger$}

\author{
Qian Yang, ${ }^{a, b}$ Chuyao Li, ${ }^{a, b}$ Jinhua Li, ${ }^{b}$ Xiaoyan Wang, ${ }^{\text {b,c }}$ Maryam Arabi, ${ }^{b}$ \\ Hailong Peng, (D) ${ }^{a}$ Hua Xiong (D) *a and Lingxin Chen (iD *b,c,d
}

Received 28th January 2020, Accepted 3rd March 2020

DOI: 10.1039/d0nr00765j

rsc.li/nanoscale

\begin{abstract}
Multiple emissions of blue, green and red from a molecular imprinting sensor rationally constructed, were used for the fluorescence colorimetric visualization of a fluorescent analyte of folic acid, using a flexible post-imprinting mixing strategy. That is, two kinds of folic acid-templated molecularly imprinted polymers (MIPs) were firstly synthesized by encapsulating green and red fluorescent quantum dots (g-QDs and $r$-QDs) individually on $\mathrm{SiO}_{2}$ cores, and they were then mixed at an appropriate ratio, resulting in a triple emission MIPs sensor. Upon folic acid recognition, the inherent blue fluorescence of folic acid was intensified, and the green and red fluorescence of the sensor QDs were gradually quenched. The quenching rate difference between $\mathrm{g}-\mathrm{QDs}$ and $\mathrm{r}$-QDs was greatly enhanced and used to obtain a wider-range and profuse fluorescence color evolution, by investigating the influences of the QDs modifier, eluent and imprinting layer thickness in detail. Under optimal conditions, the ratiometric intensity change of the three color emissions varied in a logistic function within $0.01-50 \mathrm{ppm}$ of folic acid, and the corresponding fluorescence colors shifted from yellow to orange to red to purple and finally to blue. This excellent visualization capability of the MIPs sensor contributed to the accurate naked-eye detection of folic acid concentration using a portable ultraviolet lamp. Moreover, the MIPs sensor succeeded in determining folic acid in complicated food and serum samples, providing comparable results with the PRC standard method and satisfactory recoveries of $99.5-108.0 \%$. The merits, including construction simplicity, high sensitivity and selectivity, and result visualization, enable such a multiple emission MIPs sensing strategy to be potentially applicable for visual identification and determination of various analytes in more fields.
\end{abstract}

\section{Introduction}

Molecularly imprinted polymers (MIPs) are known to possess desirable rebinding abilities by mirroring the imprinted cavities of complementary targets in terms of shape, size and functional groups. MIPs also have other unique features including remarkable physical/chemical robustness, straightforward preparation and low cost, especially compared with natural ligands such as antibodies. ${ }^{1}$ In various sensing fields, the specific analyte

\footnotetext{
${ }^{a}$ State Key Laboratory of Food Science and Technology, Nanchang University, Nanchang 330047, China. E-mail: huaxiong100@126.com

${ }^{b}$ CAS Key Laboratory of Coastal Environmental Processes and Ecological Remediation, Research Center for Coastal Environmental Engineering and Technology, Yantai Institute of Coastal Zone Research, Chinese Academy of Sciences, Yantai 264003, China.E-mail: jhli@yic.ac.cn, lxchen@yic.ac.cn

${ }^{c}$ School of Pharmacy, Binzhou Medical University, Yantai 264003, China

${ }^{d}$ Center for Ocean Mega-Science, Chinese Academy of Sciences, Qingdao 266071, China

$\dagger$ Electronic supplementary information (ESI) available. See DOI: 10.1039/ d0nr00765j
}

rebinding behavior of MIPs is transduced into measurable signals by joining them with other technologies, such as electrochemistry, ${ }^{2,3} \quad$ piezoelectricity, ${ }^{4}$ Raman, ${ }^{5}$ UV-Vis absorption,,$^{6,7}$ fluorescence, ${ }^{8,9}$ etc., to determine the analyte concentration. Among these, ratiometric fluorescence based MIPs sensors have been extensively constructed and applied, which can yield a spectrum with two or more detection signals, e.g., fluorescence emission intensities. ${ }^{10,11}$ Upon analyte recognition, the multiple fluorescence intensities are changed at different trends or degrees (e.g., enhancing/decreasing, or fast quenching/slow quenching), providing self-calibration quantitative determination, and overcoming interferences introduced by various analyte-independent factors such as instrumental fluctuation, sensing microenvironment, background fluorescence and personnel operation, that often exist when recording singleemission spectra. ${ }^{12}$ More importantly, the ratiometric intensity variations induce the transition of fluorescence color, helping visualize the contents of target analytes. ${ }^{10,11}$

Nowadays, most ratiometric fluorescence based MIPs sensors provide two emissions and their color composite pat- 
terns mainly involve blue mixed with red, ${ }^{13}$ blue mixed with green,${ }^{14}$ and green mixed with red ${ }^{11}$ to realize fluorescence color variation within their respective transition color of bluepurple-red, blue-cyan-green, and green-yellow-red, respectively, upon analyte recognition (Fig. S1†). Admittedly, their visualization capabilities are much higher than the "turnon"15,16 or "turn-off" ${ }^{17,18}$ fluorescence brightness variation of single-emission MIPs sensors. However, the color evolution windows are still narrow. Cai et al. ${ }^{19}$ have pointed out that the color variation ranges of dual-emission sensors (including but not limited to MIPs sensors) are expected to be enlarged by introducing one more emission, namely ternary emissions. In their research, ${ }^{19}$ upon interaction with $\mathrm{Cu}^{2+}$ ions, green fluorescent quantum dots (QDs) and red fluorescent QDs were quenched, but blue fluorescent carbon dots remained unchanged, resulting in wider color evolution from red to yellow, green, and finally blue. However, the selectivity of this tricolor sensor toward $\mathrm{Cu}^{2+}$ ions was not mentioned clearly. Inspired by this work and taking advantage of the selective recognition ability of MIPs, our group has constructed a ternary-emission MIPs sensor for bovine hemoglobin (BHb) visualization, where the blue, green and red fluorescence of 7-hydroxycoumarin, CdTe QDs, and CdTe/ZnS QDs are enhanced, quenched, and quenched upon binding BHb, respectively. ${ }^{20} \mathrm{~A}$ profuse color evolution following the greenyellow-red-purple-blue route is therefore attained. Obviously, QDs are excellent candidates for constructing MIPs sensors with multiplex emissions, because of their wide excitation spectra but narrow symmetrical emission profiles which allow the simultaneous excitation by a single wavelength and emission of several well-resolved peaks. ${ }^{21}$ Besides, the emission wavelength is controllable allowing location within a desired fluorescence window, and the cooperation of blue, green and red emissions is optimum for the construction of a triple emission sensor.

Importantly, compared with the unchanged blue emission in Cai et al.'s research, ${ }^{19}$ the blue fluorescence growing from low to high in our previous work ${ }^{20}$ is more favorable for enlarging color evolution. A visible blue image after complete quenching of the green and red fluorescence demands an adequate blue emission. If the blue fluorescence is unchanged throughout, the initial fluorescence image with unquenched green and red fluorescence as well as adequate blue fluorescence, will be difficult to present the near-green or near-red color, owing to the contribution of blue. Conversely, if the blue fluorescence is increased from a low value, the little blue color will have a negligible impact on adjusting the initial color. However, it is quite troublesome and difficult to find three suitable fluorescent substances that can respond to a target analyte in different variations, e.g., one enhancing and another two decreasing, or vice versa. Generally, three emissions are simultaneously quenched, or two emissions are quenched and another one is unchanged, but these go against attempts to realize broad and profuse color evolution. Accordingly, it can be reasonably deduced that triple emission MIPs sensors are more applicable to fluorescent analytes, because the intrinsic fluorescence of the analyte can provide one gradually increasing emission peak, which should work in unison with another two suppressed peaks of the sensor to construct a ternary-emission sensor.

Hence, as a proof of concept, we develop a triple emission MIPs sensor for naked-eye detection of a fluorescent analyte by a fluorescence colorimetric method, taking folic acid as the model analyte. Folic acid, biosynthesized in plant tissue, plays an essential role in the synthesis of purines, pyrimidines and DNA, as well as in cell replication in animals and humans. ${ }^{22,23}$ Inadequate folic acid intake raises the risk of cardiovascular exacerbation, malformation and neural tube defects during pregnancy, while excess ingestion can mask vitamin B-12 deficiency so as to predispose older adults to additional neuropathies. ${ }^{22}$ Thus, the concentration measurement/monitoring of folic acid is an important task. The proposed triple emission MIPs sensor was rationally constructed via a post-imprinting mixing method, i.e., the green fluorescent MIPs ( $\mathrm{g}$-MIPs) and red fluorescent MIPs (r-MIPs) were pre-synthesized separately, and then were mixed in appropriate volume proportions. The key points for successful construction of a triple emission MIPs sensor were investigated and discussed in detail. Upon rebinding folic acid, the green and red emissions of two kinds of QDs were quenched at different rates, and the blue fluorescence of folic acid was gradually enhanced. The out-of-sync change of the three emissions triggered a profuse fluorescence variation over the yellow-orange-red-purple-blue range, offering an excellent tool for the accurate visual detection of folic acid by ordinary personnel, even in complicated food and serum samples with the aid of a portable UV lamp, without requiring any bulky instruments or professional operation.

\section{Experimental section}

\subsection{Materials and reagents}

Folic acid, methotrexate (MTX), trimethoprim (TMP), thiamine chloride (VB1), ascorbic acid (VC), glycine (Gly) and glucose (Glu) were purchased from Solarbio (Beijing, China). 3-Aminopropyltriethoxysilane (APTES) and tetraethyloxysilane (TEOS) were offered by Aladdin (Shanghai, China). Ethanol (EtOH), methanol (MeOH), acetonitrile (ACN) and ammonium hydroxide $\left(\mathrm{NH}_{3} \cdot \mathrm{H}_{2} \mathrm{O}\right)$ were supplied by Sinopharm Chemical Reagent Co. Ltd (Shanghai, China). All chemicals were at least of analytical grade and were used as received. Ultrapure water of 18.2 M $\Omega$ specific resistance (Millipore, Bedford, MA, USA) was used throughout all experiments.

\subsection{Apparatus}

Fluorescence emission spectra and UV-Vis absorption spectra were recorded by a fluorescence spectrophotometer (F-7000, HITACHI) and a UV-Vis spectrophotometer (Tu-1900, PERSEE), respectively. Morphology evaluation was performed by a transmission electron microscope (TEM, JEM-2100). Elemental analysis was carried out on an energy dispersive spectrometer (EDS, INCA X-Act, Oxford). Fourier-transform infrared (FT-IR) 
spectra were obtained on an FT-IR spectrometer (Nicolet 5700, Thermo Nicolet Corp.). Size distribution was analyzed by dynamic light scattering (DLS) on a Malvern Zetasizer NanoZS90 (ZEN3590).

\subsection{Synthesis of green-emission and red-emission folic acid- templated MIPs}

Firstly, $\mathrm{SiO}_{2}$ nanoparticles, GSH-modified green fluorescent QDs (g-QDs) and MPA-modified red fluorescent QDs (r-QDs) were synthesized as previously reported, ${ }^{24,25}$ with necessary modification (synthesis details are given in the ESI†). Then, the green-emission folic acid-imprinted polymers (g-MIPs) with core-shell structure were prepared by coating the imprinted layer on the surface of $\mathrm{SiO}_{2}$ nanoparticles through a sol-gel reaction. In brief, $1 \mathrm{~mL}$ of $\mathrm{SiO}_{2}$ nanoparticles $(10 \mathrm{mg}$ $\mathrm{mL}^{-1}$ ) and $3 \mathrm{~mL}$ of $\mathrm{g}$-QDs were first dispersed in $16 \mathrm{~mL}$ of ultrapure water, and then $8 \mathrm{mg}$ of folic acid (template molecule) and $37 \mu \mathrm{L}$ of APTES were added and the solution was stirred for $1 \mathrm{~h}$. Afterwards, $50 \mu \mathrm{L}$ of $\mathrm{NH}_{3} \cdot \mathrm{H}_{2} \mathrm{O}$ and $50 \mu \mathrm{L}$ of TEOS were injected into the mixture, reacting for $12 \mathrm{~h}$ in the dark. Finally, the product (g-MIPs) was collected by centrifugation and washed with $\mathrm{MeOH}$ to remove the template folic acid completely. For comparison, green-emission nonimprinted polymers ( $\mathrm{g}$-NIPs) were also prepared by the same method but without adding folic acid. Meanwhile, red-emission folic acid-imprinted polymers (r-MIPs) and their corresponding r-NIPs were synthesized in accordance with the preparation procedures of g-MIPs and g-NIPs, respectively, but replacing g-QDs with $6 \mathrm{~mL}$ of r-QDs, reducing the volume of water to $13 \mathrm{~mL}$, increasing the volume of both $\mathrm{NH}_{3} \cdot \mathrm{H}_{2} \mathrm{O}$ and TEOS to $200 \mu \mathrm{L}$, and eluting the folic acid with ACN.

\subsection{Construction of the triple emission MIPs sensor and the fluorescence detection procedure}

For optimal visualization of folic acid, the triple emission MIPs sensor was constructed by mixing $50 \mu \mathrm{L}$ of g-MIPs and $35 \mu \mathrm{L}$ of r-MIPs in $100 \mu \mathrm{L}$ of phosphate buffer $(0.1 \mathrm{M}, \mathrm{pH} 8)$, with the final volume brought to $1 \mathrm{~mL}$ using ultrapure water. After spiking with folic acid at the final concentration of 0-50 ppm and incubating for $7 \mathrm{~min}$, the fluorescence spectra were recorded under $365 \mathrm{~nm}$ excitation with slit widths of 10/ $10 \mathrm{~nm}$. The corresponding fluorescence images were observed under a $365 \mathrm{~nm}$ UV lamp. In addition, the fabricated sensor was also used for the detection of the analogue compounds (e.g., MTX and TMP) and other substances (e.g., VB1, VC, Gly and Glu), and their corresponding changes of fluorescence spectra and images were compared with that of folic acid, where all these analytes were at the same concentration of 5 ppm.

\subsection{Analysis of real samples}

The practical use of the constructed triple emission MIPs sensor for folic acid determination was investigated in several typical real samples, involving milk powder (Yili group Co. Ltd, Inner Mongolia, China), folic acid tablets (Zhongzhou Pharma Co. Ltd, Yantai, China) and porcine serum (SenBeiJia
Biological Technology Co., Ltd, Nanjing, China). The milk powder and folic acid tablets were pretreated according to the PRC standard method GB 5009.211-2014, with details given in the ESI. $\uparrow$ The porcine serum was filtered through $0.22 \mu \mathrm{m}$ pore size membrane and diluted 100 -fold to conduct the recovery experiments at three spiked concentration levels of $0.1,5$ and $20 \mathrm{ppm}$ folic acid. Proper volumes of aqueous samples were used for assaying the folic acid contents using the constructed MIPs sensor, ensuring that the final folic acid concentrations were within the detection range. All tests were performed in triplicate, and the results were compared with those measured by the PRC standard method (GB 5009.211-2014).

\section{Results and discussion}

\subsection{Construction, characterization and possible detection mechanism of the triple emission MIPs sensor}

Fig. 1 illustrates the preparation procedures of g-MIPs and r-MIPs, the construction of the triple emission MIPs sensor, and the sensing method for the fluorescence detection of folic acid. As can be seen in route (i), g-MIPs and r-MIPs were separately synthesized by anchoring a folic acid-imprinted layer on the surface of $\mathrm{SiO}_{2}$ nanoparticles, using g-/r-QDs, folic acid, APTES, TEOS, and $\mathrm{NH}_{3} \cdot \mathrm{H}_{2} \mathrm{O}$ as the fluorescence provider, imprinting template, functional monomer, cross-linker and catalyst, respectively. After template removal, g-MIPs and r-MIPs with customized imprinted cavities complementary to folic acid in shape, size, and functional groups, were successfully obtained, and the contained g-QDs and r-QDs provided green and red fluorescence at $540 \mathrm{~nm}$ (Fig. 2A, a) and $644 \mathrm{~nm}$ (Fig. 2A, b), respectively. Besides the fluorescence emission, the presence of additional elements of S, Cd and Te in g-MIPs and r-MIPs compared with $\mathrm{SiO}_{2}$ nanoparticles (Fig. S2 $\dagger$ ), also demonstrated the successful embedding of MPA-capped g-QDs and GSH-modified r-QDs in the imprinted layer. FT-IR spectra were recorded to observe the functional groups of $\mathrm{SiO}_{2}$ nanoparticles, g-MIPs/NIPs and r-MIPs/NIPs (Fig. 2B). As can be seen, g-MIPs/NIPs and r-MIPs/NIPs had similar major backbones with related peaks at 461, 793 and $1095 \mathrm{~cm}^{-1}$, where the first two were ascribed to the symmetrical stretching vibration

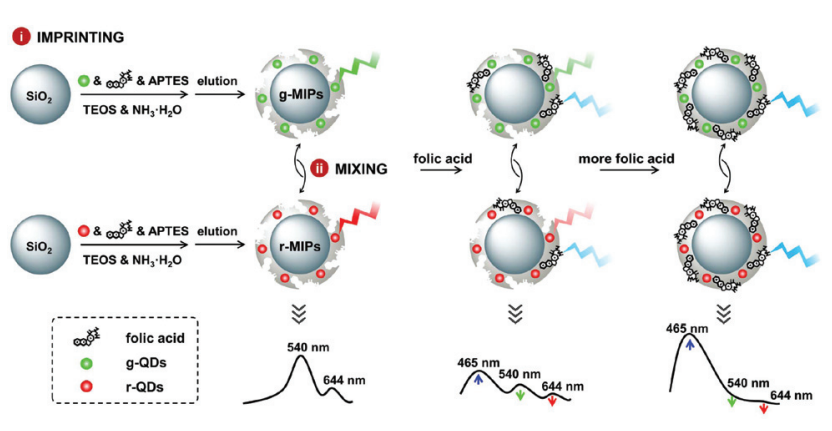

Fig. 1 Scheme illustration for (i) the synthesis procedures of g-MIPs and r-MIPs, and (ii) the construction strategy of the triple emission MIPs sensor and its application for folic acid determination. 

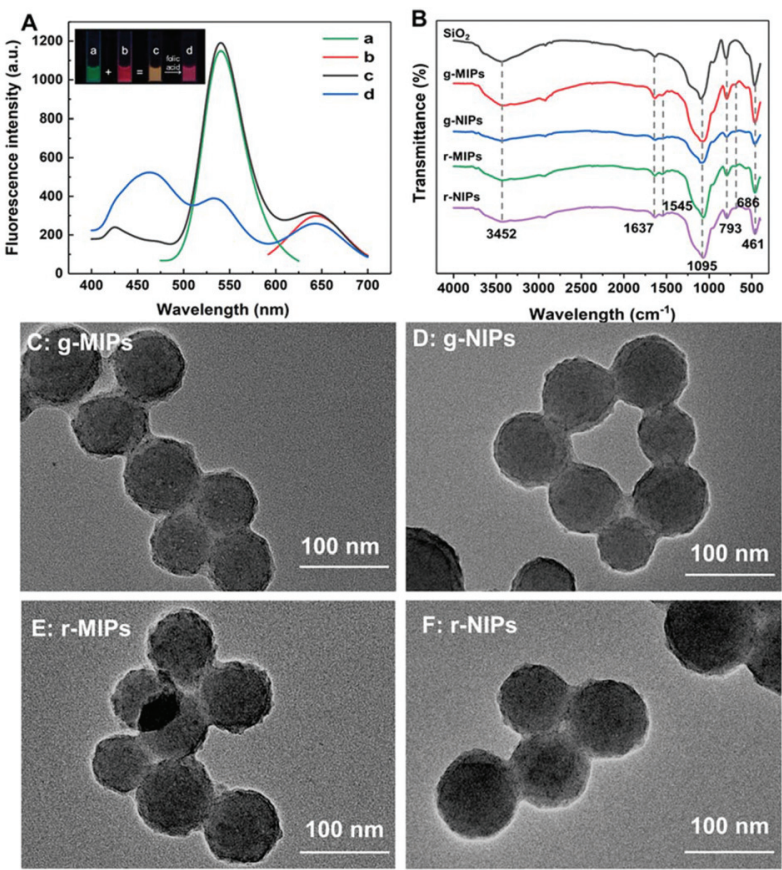

Fig. 2 (A) Fluorescence emission spectra of (a) g-MIPs, (b) r-MIPs, and the developed MIPs sensor (i.e., a mixture of $r$-MIPs and g-MIPs) (c) before, and (d) after the addition of $5 \mathrm{ppm}$ folic acid. The inset shows the corresponding fluorescence images taken under a $365 \mathrm{~nm}$ UV lamp: (B) FT-IR spectra of $\mathrm{SiO}_{2}$ nanoparticles, g-MIPs, g-NIPs, r-MIPs and r-NIPs; and TEM images of (C) g-MIPs, (D) g-NIPs, (E) r-MIPs and (F) $r$-NIPs.

of $\mathrm{Si}-\mathrm{O}$ and the last one was assigned to the antisymmetric stretching vibration of $\mathrm{Si}-\mathrm{O}-\mathrm{Si},{ }^{26}$ revealing the primary compositions of the $\mathrm{SiO}_{2}$ matrix. The characteristic peaks at $3452 \mathrm{~cm}^{-1}$ were rooted in the hydroxide of $\mathrm{SiO}_{2}$ nanoparticles and the polymeric shell, and the primary amines of APTES and GSH. The specific absorption peaks in MIPs/NIPs at 686 and $1545 \mathrm{~cm}^{-1}$ belonged to $\mathrm{C}-\mathrm{S}$ bonds and the carboxylic acid groups of GSH and MPA, ${ }^{27}$ again confirming the existence of the functional monomer of APTES, as well as the GSH-modified g-QDs and MPA-modified r-QDs in the (non-)imprinted shell. Furthermore, Fig. 2C-F display the morphologies of g-MIPs, r-MIPs and their corresponding NIPs, where MIPs and NIPs had a rougher surface than $\mathrm{SiO}_{2}$ nanoparticles (Fig. S3†), indicating the successful anchoring of the polymeric layer. The g-MIPs/NIPs and r-MIPs/NIPs had larger average diameters of approximately 85 and $93 \mathrm{~nm}$, respectively; as estimated, the polymeric layer thickness was less than $10 \mathrm{~nm}$. The ultrathin imprinting layer allowed the placement of most recognition sites in the proximity of or even at the shell surface, and therefore decreased mass transfer resistance and made the sites accessible..$^{20,28}$

After the imprinting procedure, g-MIPs and r-MIPs were well mixed at the optimal volume $(50: 35)$ to construct the triple emission MIPs sensor, defined as a "post-imprinting mixing" strategy, as schematically shown in route (ii) of Fig. 1. One fluorescence spectrum with two well-resolved emission peaks at 540 and $644 \mathrm{~nm}$ was recorded under $365 \mathrm{~nm}$ excitation, corresponding to a yellow fluorescence image (Fig. 2A, c). With the addition of folic acid, the MIPs sensor with the tailor-made binding cavities captured and rebound it, and the nearby g-QDs and r-QDs emissions were gradually quenched by the rebound folic acid at different rates, while the blue fluorescence of folic acid at $465 \mathrm{~nm}$ was enhanced (Fig. 2A, d). Thus, the ratiometric intensity of $I_{644} \times I_{540} / I_{465}$ was regularly decreased by increasing folic acid concentration, where $I_{644}$, $I_{540}$ and $I_{465}$ are the fluorescence emission intensities at 644, 540 and $465 \mathrm{~nm}$, respectively. The fluorescence image thereupon evolved from the original yellow into orange, red, purple and finally blue, providing accurate quantitative detection of folic acid with the naked eye.

Reasonably, the fluorescence decline of g-QDs and r-QDs did not stem from the fluorescence resonance energy transfer (FRET) mechanism, because no spectral overlap existed between the absorbance of folic acid and the emission of g-QDs/r-QDs (Fig. S4A $\dagger$ ). Inspired by the former studies, ${ }^{29,30}$ it was speculated that the photoinduced electron transfer (PET) process from QDs to folic acid could account for the declining fluorescence intensities. As schematically shown in Fig. S4B, $\dagger$ in the presence of folic acid, the hydrogen bond forces were formed between the hydroxyl and amino groups of folic acid and the amino groups of APTES in MIPs. ${ }^{29}$ The rebound folic acid has a UV absorption peak close to the band gap of g-MIPs and r-MIPs (Fig. S4A $\dagger$ ), so the excited charge at the conduction bands of the QDs was easily transferred to the lowest unoccupied molecular orbital of folic acid, ${ }^{30}$ as shown in Fig. S4C. $\dagger$ In this case, the process of returning the QDs' excited charge to its valence band disappeared, and therefore no fluorescence was emitted. Hence, folic acid could be quantitatively and visually detected by the developed multi-emission MIPs sensor.

\subsection{Conditions exploration for constructing the optimal triple emission MIPs sensor}

In this experiment, green and red fluorescence decreased with the addition of the target, and it is known that the synchronous intensity changes (simultaneous quenching or enhancing) at the same rate in a triple emission system would cause a narrow color variation. ${ }^{19,20}$ It means that only when there was an obvious difference in their quenching rates, could the MIPs sensor provide broader-range and profuse color evolution. Besides, it is also necessary to seek a proper intensity ratio of green- and red-emission peaks. Hence, the two key points were explored as below, on the prerequisite of optimized $\mathrm{pH}$ of 8 (Fig. S5A $\dagger$ ) and an incubation time of $7 \mathrm{~min}$ (Fig. S5B $\dagger$ ).

Firstly, the quenching rates were remarkably distinguished by increasing the green emission's quenching rate but suppressing the red emission's rate, which could be realized by optimizing three experimental parameters, namely: the modifier of the QDs, the eluent, and the thickness of the imprinting layer. As can be seen in Fig. S6, $\uparrow$ the quenching degrees (i.e., $\left.\left(I_{0}-I\right) / I_{0}\right)$ of green and red emissions were affected by the type of QD modifier (GSH, MPA, and TGA) and eluent (MeOH, 
EtOH/ACN, $\mathrm{ACN} / \mathrm{H}_{2} \mathrm{O}$, and $\mathrm{ACN}$ ). For the green emission, the quenching degree of g-QDs using GSH as the modifier greatly exceeded that of g-QDs using MPA and TGA as the modifier (Fig. S6A-C $\dagger$ ) with the same concentration of folic acid and the same eluent. With the GSH-modified g-QDs, elution using $\mathrm{MeOH}$ won out (Fig. S6A $\dagger$ ). The quenching degrees of g-MIPs, embedded with GSH-modified g-QDs and eluted by $\mathrm{MeOH}$, were up to $20.41 \%$ and $69.06 \%$ at 0.5 and $10 \mathrm{ppm}$, respectively (Fig. S6A $\dagger$ ). Moreover, in a similar manner, the degree of red emission quenching affected by the modifier and eluent was also investigated: distinctly, the recognition of folic acid by MPA-modified r-QDs was greatly inferior to that of GSH- and TGA-modified r-QDs (Fig. S6D-F $\dagger$ ); and with the same modifier of MPA, r-MIPs displayed a lower quenching rate using ACN as the eluent - less than $0.24 \%$ and $18.40 \%$ at 0.5 and $10 \mathrm{ppm}$ folic acid, respectively (Fig. S6E†). Hence, GSH-modified $\mathrm{g}$-QDs and MPA-capped r-QDs were embedded in the g-MIPs and r-MIPs, using $\mathrm{MeOH}$ and ACN to remove folic acid, respectively.

In addition, more effort was paid to further expand the quenching rate difference, such as by changing the thickness of the imprinting layer. Many published studies have confirmed that a thicker imprinted shell goes against the sensitive determination of analytes, and the thickness can be controlled by adjusting the polymerization method, imprinting time, reagent dosage, etc. ${ }^{31-33}$ Herein, the imprinting shell thickness of r-MIPs was controlled by adjusting the amount of crosslinker (TEOS) and catalyst $\left(\mathrm{NH}_{3} \cdot \mathrm{H}_{2} \mathrm{O}\right)$. As revealed in the size distribution results measured by DLS (Fig. S7A-E $\dagger$ ), the average size of $\mathrm{SiO}_{2}$ was about $90 \mathrm{~nm}$ and several r-MIPs were approximately 96, 100, 110 and $118 \mathrm{~nm}$ corresponding to different dosages of TEOS and $\mathrm{NH}_{3} \cdot \mathrm{H}_{2} \mathrm{O}$ at 50, 100, 200 and $300 \mu \mathrm{L}$, respectively, indicating that the imprinting layer was getting thicker with increasing amounts of TEOS and $\mathrm{NH}_{3} \cdot \mathrm{H}_{2} \mathrm{O}$. Then, the quenching degree of r-MIPs, prepared with increasing TEOS and $\mathrm{NH}_{3} \cdot \mathrm{H}_{2} \mathrm{O}$ volume (equal to growing MIPs shell thickness), exhibited gradual decline and reached an equilibrium at $200 \mu \mathrm{L}$ (Fig. S7F $\dagger$ ). It was interpreted that the imprinted cavities were deeply encapsulated in the polymer network with a higher cross-linker degree, hindering template removal and target adsorption and lessening the number of specific binding sites per unit mass of MIPs, ${ }^{1,34}$ and therefore the quenching effect was lowered. Hence, to further suppress the quenching rate of r-MIPs, $200 \mu \mathrm{L}$ of TEOS and $\mathrm{NH}_{3} \cdot \mathrm{H}_{2} \mathrm{O}$ was used for anchoring the r-MIPs shell on the $\mathrm{SiO}_{2}$ core.

After the design of g-MIPs and r-MIPs by the aforementioned specific methods, their volume ratio in the MIPs sensor (total volume: $1 \mathrm{~mL}$ ) was further investigated, which determined the original intensity ratio of green and red emissions. As illustrated in Fig. 3A, the MIPs sensor consisting of only 5\% g-QDs (i.e., $50 \mu \mathrm{L}$ ), displayed a gradually reducing green-emission peak and growing blue fluorescence with folic acid addition, with a narrow color shift from green to blue. When the volume fraction of r-MIPs increased from $0.5 \%$ to $8 \%$ (i.e., $5-80 \mu \mathrm{L}$ ) with a fixed composition of $5 \%$ of $\mathrm{g}$-MIPs, it was observed that the red fluorescence peak was heightened
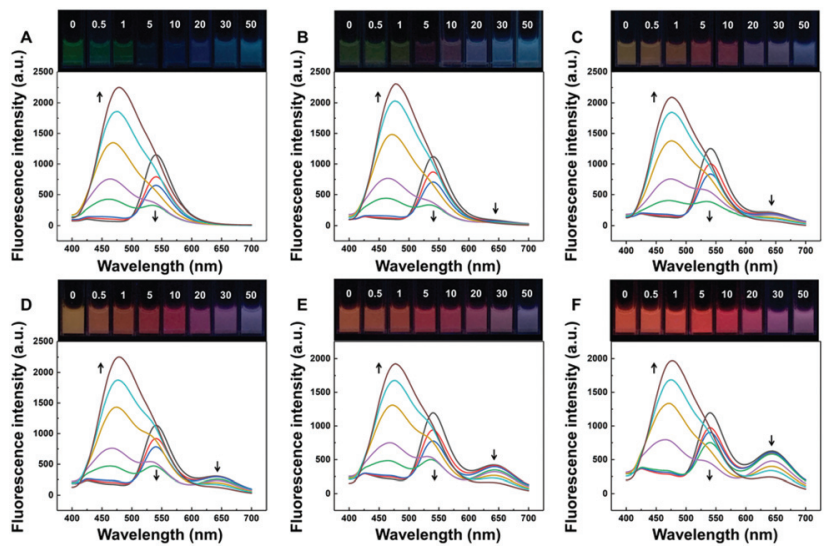

Fig. 3 Fluorescence spectra and images of MIPs sensor toward 0-50 ppm folic acid, where the MIPs sensor was composed of $50 \mu \mathrm{L}$ of g-QDs and (A) 0, (B) 5, (C) 20, (D) 35, (E) 50 or (F) $80 \mu \mathrm{L}$ of $r$-QDs, respectively.

(Fig. 3B and F) and the initial color of the as-obtained MIPs sensor obviously changed from olive green to yellow to orangered, with brightness improving simultaneously (upper of Fig. 3). However, the MIPs sensors with an extremely low amount of r-MIPs $(0.5 \%)$ failed to provide a profuse color without the appearance of a red segment (Fig. 3B); and the yellow part was missed with excessive use of r-MIPs of $5 \%$ and 8\% (Fig. 3E and F). Excitingly, when 5\% g-MIPs were combined with $2 \%$ or $3.5 \%$ r-MIPs, an ideal wide-ranging fluorescence color evolution of yellow-orange-red-purple-blue occurred (Fig. 3C and D). Then, in comparison to the MIPs sensor consisting of $2 \%$ r-MIPs (Fig. 3C), the MIPs sensor with 3.5\% r-MIPs was endowed with a relatively brighter image, as well as more delicate and profuse fluorescence variation before and after the red inflection point (at $5 \mathrm{ppm}$ folic acid, Fig. 3D). Therefore, the MIPs hybrid integrating 5\% g-MIPs and 3.5\% r-MIPs was determined as the optimal MIPs sensor for further use, promising to provide the best visual outcome.

As mentioned above, the volume ratio of $\mathrm{g}$-MIPs and r-MIPs would determine the initial color as well as the color evolution of the as-constructed MIPs sensor. With the fast quenching green emission but slow quenching red emission toward folic acid, the volume ratio of g-MIPs and r-MIPs should be large enough to obtain the initial near-green or yellow image, followed by a wide color evolution range of yellow-orange-redpurple-blue. Furthermore, it can be reasonably deduced that if red emission is quenched faster than green emission, color variation following a red-orange-yellow-green-cyan-blue route (Fig. S8 $\dagger$ ) would be provided by the MIPs sensor, where the volume ratio of g-MIPs and r-MIPs should be small enough to make the initial fluorescence color near-red.

\subsection{Multiplex and visual detection of folic acid by the triple emission MIPs sensor}

Under the above-optimized conditions, the triple emission MIPs sensor was used for the multiplex and visual detection of 

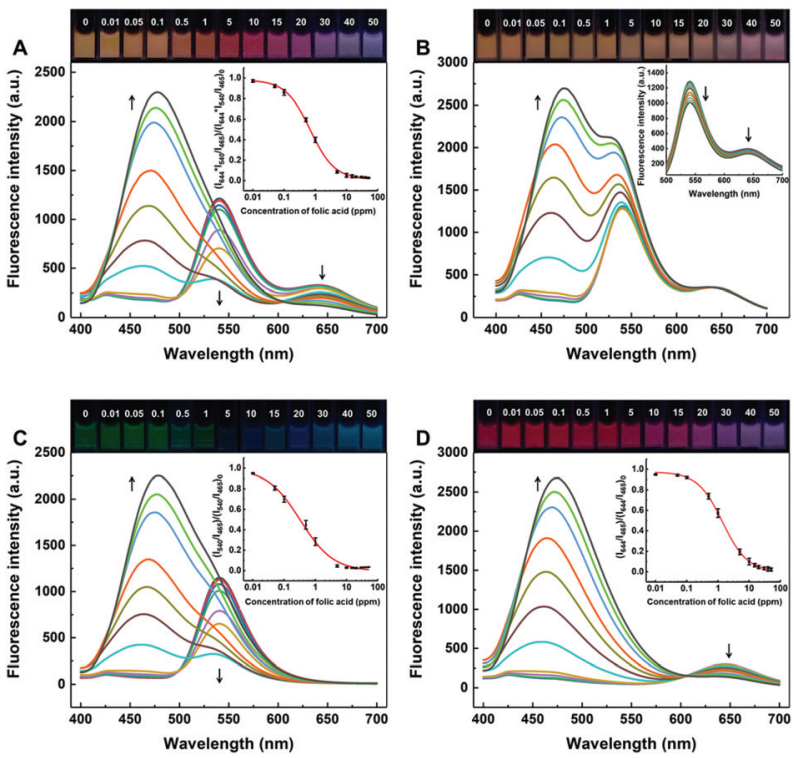

Fig. 4 Fluorescence spectra and corresponding images of (A) triple emission MIPs sensor, (B) triple emission NIPs sensor, and dual emission MIPs sensors of (C) blue- and green-emission, and (D) blue- and redemission toward 0-50 ppm folic acid. Insets of (A), (C) and (D) show the fitting curves for the ratiometric intensity changes, with related parameters given in Table $\mathrm{S} 1 ; \uparrow$ and the inset of (B) shows the fluorescence spectra of green-emission and red-emission peaks after eliminating the effect of spectral overlap from the blue-emission peak with increasing folic acid.

folic acid. As can be seen in Fig. 4A, before folic acid addition, the MIPs sensor provided only green-emission and red-emission peaks at 540 and $644 \mathrm{~nm}$ under single excitation. A yellow fluorescent image presented under a $365 \mathrm{~nm}$ UV lamp. Upon folic acid addition from 0 to $1 \mathrm{ppm}$, the fluorescence intensity of the green-emission peak was drastically dropped to half of the original value, while only a tiny decrease was observed in the red fluorescence intensity because of its deliberately limited quenching rate, and almost no blue fluorescence from the folic acid itself appeared. The dominant contribution of the green-emission peak gave rise to the fluorescence color shift from yellow to orange. With folic acid concentration increasing to $5 \mathrm{ppm}$, most of the green fluorescence disappeared, a small amount of r-QDs were quenched, and the blue fluorescence peak from 5 ppm folic acid appeared but had little impact. So, the fluorescence color continued to look red, easily observed by the naked eye. With further addition of folic acid to $50 \mathrm{ppm}$, the impact of the further diminishing green fluorescence was limited but the r-QDs were almost completely quenched, and the blue fluorescence was regularly enhanced. The dynamic regulation of the dimming red fluorescence and increasing blue fluorescence resulted in the further color change to purple and finally to blue. The ratiometric intensity change of red-, green- and blue-emission peaks, i.e., $\left(I_{644} \times I_{540} / I_{465}\right) /\left(I_{644} \times I_{540} / I_{465}\right)_{0}$, obeyed a logistic function (inset of Fig. $4 \mathrm{~A}$ and Table $\mathrm{S} 1 \dagger$ ) with a high correlation coefficient $\left(r^{2}\right)$ of 0.9988 , where $\left(I_{644} \times I_{540} / I_{465}\right)_{0}$ and
$\left(I_{644} \times I_{540} / I_{465}\right)$ mean the ratiometric intensity of three emission peaks before and after folic acid addition. An ultralow detection limit of $0.0052 \mathrm{ppm}$ folic acid was obtained based on the $3 \sigma$ IUPAC rule.

In contrast, the triple emission NIPs sensor exhibited enhancing blue and green fluorescence and unaffected red fluorescence with folic acid addition (Fig. 4B). It was easy to understand the folic acid-triggered increase of blue fluorescence, but the raised green fluorescence was quite incomprehensible. After eliminating the effect of spectral overlap from the blue-emission peak, it was found that both greenand red-emission peaks were quenched by increasing folic acid, but weakly (inset of Fig. 4B). With the distinct blue fluorescence enhancing, but the slight decreasing of green and red fluorescence, the fluorescence color of the NIPs sensor did not change from yellow to pink-purple until folic acid concentration increased to higher than $30 \mathrm{ppm}$ (upper of Fig. 4B). Comparing the analysis performance (both the spectra and image changes) of the MIPs sensor, we were convinced that there were numerous specific cavities playing the recognition role in the imprinting layer, and the tiny intensity alteration of the NIPs sensor was attributed to the nonspecific adsorption.

The analysis performance of the triple emission MIPs sensor was further compared with that of dual emission MIPs sensors. As shown in Fig. 4C and D, similarly, the green-emission and red-emission peaks were quenched, and the blue emission was enhanced upon folic acid addition, resulting in ratiometric intensity changes with a similar trend (insets of Fig. 4C and D, and Table S1 $\dagger$ ). Compared with the slopes of the fitting curves, it was discovered that the ratiometric intensity change of the triple emission MIPs sensor (inset of Fig. 4A) was less sensitive than that of the green- and blueemission MIPs sensor (inset of Fig. 4C) when folic acid was at trace concentrations, because the r-MIPs competitively bound some target folic acid molecules but an intensity change in emission caused by this behavior was rare. On the contrary, the red- and blue-emission MIPs sensor (inset of Fig. 4D) was no match for the triple emission, because the green fluorescence was much more sensitive towards trace folic acid than the red. Owing to their just being a dual peaks change of rising blue fluorescence and declining green/red fluorescence, color variation was absolutely compressed in the green-blue or red-purple scope (upper of Fig. 4C and D). Moreover, for the single emission MIPs sensor, it was conceivable that only the higher blue fluorescence intensity and brighter blue image could be observed. To sum up, the triple emission MIPs sensor was significantly better than all the single and dual emission MIPs sensors, especially the capacity to provide a broad-range and profuse fluorescence color evolution for realizing the accurate analyte visualization.

\subsection{Selectivity and stability evaluation of the MIPs sensor}

To appraise the selectivity of the tricolor MIPs sensor, some analogue compounds such as MTX and TMP as well as some other possible concomitant substances like VB1, VC, Gly and Glu were investigated. Firstly, folic acid was recognized by the 
imprinted cavities (complementary in shape, size and functional groups) of the MIPs sensor and therefore quenched the emission of the g-QDs and r-QDs, so the ratiometric intensity of $I_{644} \times I_{540} / I_{465}$ was diminished and the fluorescent color turned red (Fig. 5, upper-left). However, most other substances except MTX had negligible influence on the ratiometric intensity change with the same unobservable color change (Fig. 5, upper-left). It revealed that the folic acid-imprinted cavities were not complementary to other molecules so no rebinding behavior occurred, but MTX has a similar structure to folic acid so a small part of MTX was rebound and caused a certain amount of quenching of the QDs emission. Meanwhile, the NIPs sensor had no specific recognition site toward any molecule so the embedded g-QDs and r-QDs were not affected by folic acid and other substances; however, folic acid emitted blue fluorescence with a certain intensity, leading to an obvious ratiometric intensity change (Fig. 5). Fortunately, the insufficient blue fluorescence made little difference to the fluorescence color of the NIPs sensor, with similar fluorescence as shown in the images toward other substances (upper-right of Fig. 5).

Additionally, the stability of the tricolor MIPs sensor was investigated by repeatedly measuring the fluorescence spectra

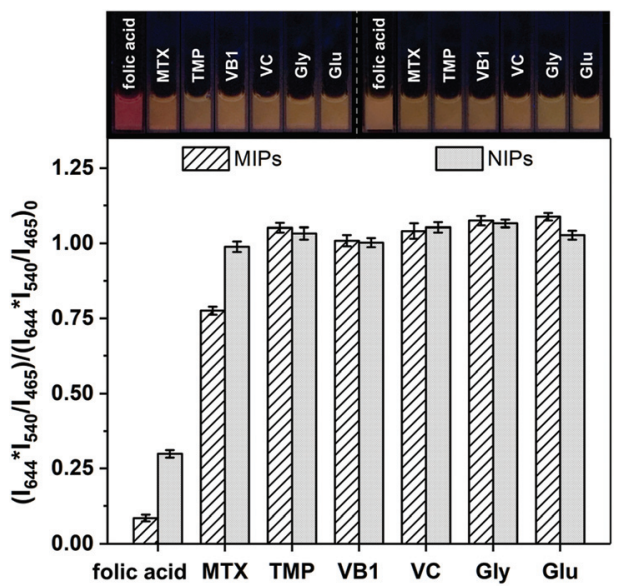

Fig. 5 Ratiometric intensity changes of MIPs sensor and NIPs sensor toward folic acid, MTX, TMP, VB1, VC, Gly and Glu at the same concentration of 5 ppm. Upper: the corresponding fluorescence images of MIPs sensor (left) and NIPs sensor (right). every day before and after interaction with $5 \mathrm{ppm}$ folic acid. As displayed in Fig. S9, $\uparrow$ the intensities of green and red emissions almost remained constant during the 10 days storage. Meanwhile, the ratiometric intensity change for $5 \mathrm{ppm}$ folic acid remained stable without obvious change. Both facts confirmed that the developed MIPs sensor possessed excellent storage stability without a significant decrease in its sensing performance.

\subsection{Practical applicability of the MIPs sensor}

Based on the extraordinary selectivity, sensitivity and stability, the developed MIPs sensor was used for quantifying folic acid in three different real samples, with the tested results shown in Table 1. As can be seen, folic acid concentration in milk powder tested with the MIPs sensor was $158 \mu \mathrm{g}$ per $100 \mathrm{~g}$, being highly consistent with that $(167 \mu \mathrm{g}$ per $100 \mathrm{~g})$ tested by the microbiological method given in the PRC standard of GB 5009.211-2014. And folic acid content in tablets was $0.385 \mathrm{mg}$ per tablet and $0.408 \mathrm{mg}$ per tablet detected by the MIPs sensor and the microbiological method, respectively, showing the low deviation of $-3.75 \%$ and $2 \%$ against the labeled value of $0.40 \mathrm{mg}$ per tablet. The tested results demonstrated that folic acid content was within the authorized deviation range $( \pm 15 \%)$ given in the PRC standard of GB 28050-2011. In addition, recovery experiments for porcine serum were performed using the MIPs sensor at three spiked concentrations, and recoveries ranging from 99.5 to $108.0 \%$ were achieved with relative standard deviations (RSDs) lower than 3.0\%. These results demonstrated the excellent practicability of the developed MIPs sensor for the detection of folic acid in complex real samples.

\section{Conclusions}

To sum up, a novel triple emission MIPs sensor was rationally constructed by a post-imprinting mixing method for multiplex ratiometric and visual determination of folic acid, providing folic acid-dependent growing blue fluorescence and decreasing green and red fluorescence, with wide-range and profuse color variation. The key variables for the successful construction were explored and optimized. The sensor benefited from the advantages of simple construction by the post-imprinting mixing strategy, outstanding selectivity and sensitivity of the

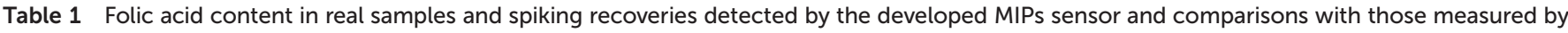
the microbiological method given in the PRC standard of GB 5009.211-2014

\begin{tabular}{|c|c|c|c|c|}
\hline Samples & Microbiological method (mean $\pm \mathrm{CV}^{a}$ ) & Spiked & MIPs sensor (mean \pm RSD (\%)) & Recovery (mean \pm RSD (\%)) \\
\hline Milk powder & $167 \mu g$ per $100 \mathrm{~g} \pm 0.8$ & 0 & $158 \mu \mathrm{g}$ per $100 \mathrm{~g}^{b} \pm 2.1$ & - \\
\hline Folic acid tablets & $0.408 \mathrm{mg}$ per tablet \pm 4.0 & 0 & $0.385 \mathrm{mg}$ per table $\mathrm{e}^{c} \pm 3.2$ & - \\
\hline \multirow[t]{4}{*}{ Porcine serum } & - & 0 & - & - \\
\hline & & $0.100 \mathrm{mg} \mathrm{L}^{-1}$ & $0.108 \mathrm{mg} \mathrm{L}^{-1}$ & $108.0 \pm 2.0$ \\
\hline & & $5.000 \mathrm{mg} \mathrm{L}^{-1}$ & $5.147 \mathrm{mg} \mathrm{L}^{-1}$ & $102.9 \pm 2.9$ \\
\hline & & $20.000 \mathrm{mg} \mathrm{L}^{-1}$ & $19.905 \mathrm{mg} \mathrm{L}^{-1}$ & $99.5 \pm 1.6$ \\
\hline
\end{tabular}

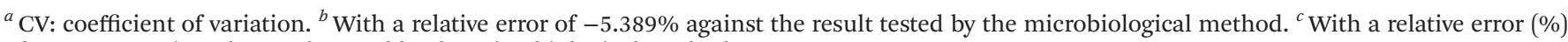
of $-5.637 \%$ against the result tested by the microbiological method. 
molecular imprinting fluorescence detection, and the ideal coordination between blue-, green- and red-emission peaks. More prominently, the color evolution range covered the yellow-orange-red-purple-blue window, making for more reliable and accurate naked-eye detection of target contents even in complicated real samples. Also, the unparalleled visualization capability suits perfectly non-specialised users with a portable UV lamp, so there is no need for well-trained personnel or large complex instruments. We expect that this successful proof of concept would accelerate the development of multiplex-emission MIPs sensors and their wide applications in more fields, especially for on-site visual determination of fluorescent analytes.

\section{Conflicts of interest}

There are no conflicts to declare.

\section{Acknowledgements}

This work was financially supported by the National Natural Science Foundation of China (21667018, 21876199, 31860470, 21804010, 21976209, 31660482), the Science and Technology Development Plan of Yantai City of China, the Chinese Academy of Sciences President's International Fellowship Initiative (2019PC0050) and the Taishan Scholar Project Special Funding (ts20190962).

\section{References}

1 L. Chen, X. Wang, W. Lu, X. Wu and J. Li, Chem. Soc. Rev., 2016, 45, 2137-2211.

2 D. Udomsap, C. Branger, G. Culioli, P. Dollet and H. Brisset, Chem. Commun., 2014, 50, 7488-7491.

3 N. A. El Gohary, A. Madbouly, R. M. El Nashar and B. Mizaikoff, Biosens. Bioelectron., 2015, 65, 108-114.

4 H. F. EL-Sharif, H. Aizawa and S. M. Reddy, Sens. Actuators, $B, 2015,206,239-245$.

5 J. Ye, Y. Chen and Z. Liu, Angew. Chem., Int. Ed., 2014, 53, 10386-10389.

6 J. Deng, S. Chen, J. Chen, H. Ding, D. Deng and Z. Xie, ACS Appl. Mater. Interfaces, 2018, 10, 34611-34617.

7 Z. Wu, X. Hu, C. Tao, Y. Li, J. Liu, C. Yang, D. Shen and G. Li, J. Mater. Chem., 2008, 18, 5452-5458.

8 K. Mori, M. Hirase, T. Morishige, E. Takano, H. Sunayama, Y. Kitayama, S. Inubushi, R. Sasaki, M. Yashiro and T. Takeuchi, Angew. Chem., Int. Ed., 2019, 58, 1612-1615.

9 M. Sobiech, P. Bujak, P. Lulinski and A. Pron, Nanoscale, 2019, 11, 12030-12074.

10 Q. Yang, J. Li, X. Wang, H. Peng, H. Xiong and L. Chen, Biosens. Bioelectron., 2018, 112, 54-71.
11 Q. Yang, J. Li, X. Wang, H. Peng, H. Xiong and L. Chen, Sens. Actuators, B, 2019, 284, 428-436.

12 M. H. Lee, J. S. Kim and J. L. Sessler, Chem. Soc. Rev., 2015, 44, 4185-4191.

13 M. Li, H. Liu and X. Ren, Biosens. Bioelectron., 2017, 89, 899-905.

14 M. Amjadi and R. Jalili, Biosens. Bioelectron., 2017, 96, 121126.

15 Q. Li, T. Kamra and L. Ye, Chem. Commun., 2016, 52, 12237-12240.

16 A. B. Descalzo, C. Somoza, M. C. Moreno-Bondi and G. Orellana, Anal. Chem., 2013, 85, 5316-5320.

17 M. P. Chantada-Vazquez, J. Sanchez-Gonzalez, E. PenaVazquez, M. J. Tabernero, A. M. Bermejo, P. BermejoBarrera and A. Moreda-Pineiro, Biosens. Bioelectron., 2016, 75, 213-221.

18 H. Sunayama and T. Takeuchi, ACS Appl. Mater. Interfaces, 2014, 6, 20003-20009.

19 Y. Cai, J. You, Z. You, F. Dong, S. Du and L. Zhang, Biosens. Bioelectron., 2018, 99, 332-337.

20 Q. Yang, J. Li, X. Wang, H. Xiong and L. Chen, Anal. Chem., 2019, 91, 6561-6568.

21 S. Silvi and A. Credi, Chem. Soc. Rev., 2015, 44, 42754289.

22 R. J. Pawlosky and V. P. Flanagan, J. Agric. Food Chem., 2001, 49, 1282-1286.

23 N. Delchier, A. L. Herbig, M. Rychlik and C. M. G. C. Renard, Compr. Rev. Food Sci. Food Saf., 2016, 15, 506-528.

24 X. Wu, Z. Zhang, J. Li, H. You, Y. Li and L. Chen, Sens. Actuators, B, 2015, 211, 507-514.

25 M. Y. Gao, S. Kirstein, H. Mohwald, A. L. Rogach, A. Kornowski, A. Eychmuller and H. Weller, J. Phys. Chem. B, 1998, 102, 8360-8363.

26 Z. Zhou, H. Ying, Y. Liu, W. Xu, Y. Yang, Y. Luan, Y. Lu, T. Liu, S. Yu and W. Yang, Appl. Surf. Sci., 2017, 404, 188196.

27 M. Gazizadeh, G. Dehghan and M. Amjadi, Luminescence, 2019, 34, 297-303.

28 J. Bognar, J. Szucs, Z. Dorko, V. Horvath and R. E. Gyurcsanyi, Adv. Funct. Mater., 2013, 23, 47034709.

29 A. A. Ensafi, P. Nasr-Esfahani and B. Rezaei, Anal. Chim. Acta, 2017, 996, 64-73.

30 Y. Yang, Z. Wang, H. Niu and H. Zhang, Biosens. Bioelectron., 2016, 86, 580-587.

31 R. Long, T. Li, C. Tong, L. Wu and S. Shi, Talanta, 2019, 196, 579-584.

32 R. Shariati, B. Rezaei, H. R. Jamei and A. A. Ensafi, Talanta, 2019, 194, 143-149.

33 M. Peng, H. Xiang, X. Hu, S. Shi and X. Chen, J. Chromatogr. A, 2016, 1474, 8-13.

34 Y. Yan, X. He, W. Li and Y. Zhang, Biosens. Bioelectron., 2017, 91, 253-261. 